\title{
Die Geschichte vom gemachten Nest - und kein Vogel will sich hineinsetzen
}

Hans-Ueli Würsten

Korrespondenz:

Dr. med. Hans-Ueli Würsten

Leitender Arzt Chirurgie

Spitalzentrum Biel

Postfach 1664

CH-2501 Biel/Bienne

Tel. 0323243224

Fax 0323243209

hans-ueli.wuersten@szb-chb.ch
Es war einmal ein Krankenhaus, gelegen in einer lieblichen, aber zugegebenermassen in einer dornröschenverschlafenen Gegend, der auch ein Grossereignis wie die Expo 02 höchstens ein Räuspern und eine schwungvolle Drehung im Bett zum Weiterschlafen entlockt hatte. Touristische Möglichkeiten hätte es, Leute kommen hierher in Urlaub. Männiglich staunt über die vielen Uhren mit grossen Namen, KLEIN-e mit grossem Namen von A bis Z (zur Vermeidung von Schleichwerbung wird an dieser Stelle auf das griechische Alphabet verzichtet). Jeder scheint hier auf eine Zehntelsekunde pro 25000 Jahre genau zu wissen, wie spät es ist.

Das berühmte Krankenhaus dieser Gegend hat sich dazu entschlossen, ebenfalls im Zeichen der Zeit zu handeln und etwas für seine Grundversorgung bzw. die ach so gebeutelten Allgemeinpraktiker zu tun. Unerhörtes ist passiert: Sämtliche Kliniken haben sich auf ein umfassendes Angebot für eine Weiterbildung zum Facharzt Allgemeinmedizin einigen können! Die Botschaft ist in das Land hinausgegangen:

Wir bieten:

- 1 Jahr Chirurgie;

- 2 Jahre innere Medizin;

- 1 Jahr Gynäkologie/Geburtshilfe,

- 1 Jahr Kinderheilkunde mit Rotation in den Praxisbereich.

Darüber hinaus ist in Aussicht gestellt worden, Hilfeleistung zu bieten für die anschliessende Praxiseröffnung bzw. Praxisübernahme in der Gegend.

Die betroffenen Kliniken haben denn auch gehorsam in ihrer Planung die entsprechenden Stellen zum geforderten Zeitfenster bereitgestellt.

Erwartungsvoll hat man nun der Lawine der Bewerbungen geharrt. Aus der Lawine sind jedoch 3-4 Schneeflocken geworden.

In einem ersten Anlauf hat denn auch die Stelle erfolgreich besetzt werden können. Natürlich war für jedes neue Jahr ein neues solches Curriculum geplant. Die Bilanz im Hinblick auf die Besetzung der Stelle ab 1. Januar 2008 sieht wie folgt aus:
Offre en formation postgraduée

du Centre hospitalier Bienne

pour les médecins de premier

\section{recours}

En guise de contribution active à l'établissement d'une bonne assistance médicale de premier recours à la population, le Centre hospitalier Bienne offre depuis janvier 2007 chaque année un programme complet de formation postgraduée pour I'obtention du titre FMH en médecine générale. Par ailleurs, l'on soutient également les intéressés lors de la reprise ou de la création d'un nouveau cabinet médical dans la région. Malgré l'importance de l'offre, les demandes ont été très faibles jusqu'à présent.

- Bewerbungen aus dem deutschsprachigen EU-Raum: 4;

- Bewerbungen aus dem deutschsprachigen EU-Raum mit Abstammung ausserhalb, aber mit deutscher Ausbildung: 1;

- Bewerbungen aus dem übrigen EU-Raum: 0;

- Bewerbungen aus der Schweiz von Schweizern mit Schweizer Ausbildung: 0.

Es kommen dazu 2 Bewerbungen von Kandidatinnen, die schon über ein praktisch vollständiges Weiterbildungsprogramm verfügen und deswegen nicht in Betracht gezogen werden konnten. Eine wahrlich reiche Ernte ...

- Ist der Beruf des Grundversorgers derart unattraktiv geworden, dass selbst massgeschneiderte Angebote, die potentiell einen Karrierepfad für das Leben bedeuten, nicht mehr attraktiv sind?

- Führen die effektvollen, selbstbemitleidenden Kampagnen der Grundversorger selbst dazu, dass die jungen Leute von einem solchen Leben abgeschreckt werden? 
Bleibt zurück die Information vom Hörensagen, dass die Randbedingungen für Grundversorger in Deutschland offenbar noch viel schwieriger sind, so dass vergleichsweise dieser Beruf in der Schweiz wesentlich attraktiver zu sein scheint.

Müssen wir es also als Tatsache hinnehmen, dass nicht nur entlegenste Talschaften eine Zukunft der medizinischen Unterversorgung vor Augen haben werden? Dass auch wohn- und lebensattraktive Gegenden, insbesondere aber die Städte, für sogenannte «Nichtspezialisten» unattraktiv werden, so dass selbst dort die Grundversorgung und ihre ureigene medizinische Kultur bedroht sind?

Niemand stuft Zehnkämpfer niedriger ein als die Superspezialisten. Wollen sie auf hoher Stufe Bestand haben, müssen sie in zwei Disziplinen nahe an der Weltspitze sein, in mindestens 5-6 weiteren Fachdisziplinen auf nationalem Niveau mittun. $\mathrm{Zu}$ dieser Leistung ist jedem zu gratulieren, der Aufwand dazu ist sicherlich hoch. Niemand wird ernsthaft bestreiten, dass der Zehnkämpfer wohl die meisten und härtesten
Trainingseinheiten aufbringt, um das Geforderte zu leisten.

Das Anliegen der Spitäler kann es sein, hier die optimale und maximale Unterstützung zu bieten. Wenn denn nicht nur ein Weiterbildungsgang, sondern zusätzlich die Hilfeleistung zur Übernahme eines allfälligen bestehenden Patientenstammes in Aussicht steht, ist vielleicht nicht alles Mögliche, aber doch sehr viel von dieser Seite getan worden. Wie sagte doch schon die automatische Küchenmaschine: «Nur no ässe müend Sie sälber.»

Dass in dieser uhrenreichen Stadt nicht nur die Zeit wahrgenommen worden ist, sondern sogar deren Zeichen erkannt worden sind, ist den betreffenden verantwortlichen Chefärzten, die zugegebenermassen selbst Spezialisten sind, sicherlich hoch anzurechnen.

Um klar zu dokumentieren, dass das Märchen einen Wahrheitsgehalt beansprucht, fügen wir den entsprechenden Link hier an: www.jobs.ch oder www.spital-biel.ch. 